\title{
Mechanism of adhesion and graft polymerization of PNIPAAm thermoresponsive hydrogel to polypropylene meshes
}

\author{
Sonia Lanzalaco, ${ }^{\mathrm{a}, \mathrm{b}, \S}$ Pau Turon, ${ }^{\mathrm{c}}$ Christine Weis, ${ }^{\mathrm{c}}$ Carlos Alemán ${ }^{\mathrm{a}, \mathrm{b}}$ and Elaine Armelin $^{\mathrm{a}, \mathrm{b}, \S}$ \\ In this study, a commercial and fully-flexible monofilament mesh has been used for the deposition of a thermosensitive hydrogel, generated by graft \\ copolymerization of $\mathrm{N}$-isopropylacrylamide (NIPAAm) and $\mathrm{N}, \mathrm{N}$ '-methylene bis(acrylamide) (MBA) monomers. The mechanism of adhesion and graft \\ copolymerization have been elucidated combining micro-and standard spectroscopy techniques such as Raman, FTIR and XPS; before and after the activation \\ of the polypropylene (PP) fibre surface by using oxygen-plasma. The good covalent interaction among NIPAAm monomers and PP fibres, and the hydrogel \\ chain growth in such flexible bidimensional structures, were demonstrated. Additionally, the thermoresponsive properties of PNIPAAm was approached (VPTT \\ behaviour). The bilayer system is stable below and above the low critical solution temperature (LCST) of $33.2^{\circ} \mathrm{C}$.
}

Keywords: thermosensitive hydrogel, $\mathrm{N}$-isopropylacrylamide, polypropylene, surgical mesh, XPS spectroscopy

\section{Introduction}

Non-absorbable surgical meshes, which are manufactured using fibres of isotactic polypropylene (iPP), are knitted to exhibit mono- or multifilament geometries depending on their clinical intended use. Among polymers, PP is one of the most important thermoplastics employed in the biomedical field, ${ }^{1-5}$ particularly as patch repair for contact with abdominal wall, since Francis Usher, ${ }^{6,7}$ a distinguished herniologist, used it as a graft for tissue reinforcement. However, iPP is not reactive and surface functionalization is necessary for the deposition and adhesion of other biocompatible materials, like stimuli-responsive soft materials, poly( $N$-isopropylacrylamide)-based hydrogels (PNIPAAm). ${ }^{8}$

Plasma irradiation on polymer surfaces is the most widely used technique for cleaning from adsorbed contaminants and effective activation of material surfaces, even though it is also applied for other purposes, as for example etching and cross-linking. ${ }^{9}$ If properly controlled, activation of polymer surfaces by plasma exposure presents important environmental advantages with respect to standard activation methods, as for example those based on aggressive reagents or harmful radiations. ${ }^{10,11}$

Although there are several works focused on the activation of PP surface with plasma technology, ${ }^{12-15}$ the effects of plasma on PP meshes, such as monofilament threads and 2D multifilament prosthesis have been scarcely reported. As few examples, Gorgieva et al. ${ }^{12}$ studied the influence of $\mathrm{O}_{2}$-plasma activated PP surface in the adhesion of gelatin-coated mesh fibres with in situ integrated antimicrobial $\varepsilon$-poly-L-lysine immobilized on the PP-gel biomimetic scaffolds. Canal and co-workers ${ }^{16}$ reported a new strategy to functionalize and upload ampicillin antibiotic in high density PP surgical meshes. They successfully immobilized the drug between the plasma treated PP fibres and a polyethylene glycol (PEG) nanometric layer by applying both plasma functionalization and plasma polymerization.

The mechanism of chemical adhesion of polymers to solid surfaces, under plasma activation, depends on several factors, such as: $(i)$ plasma equipment and geometry of chamber; ${ }^{17} 18$ (ii) chemical gases used in plasma equipment (oxygen, argon, atmospheric, etc.); ${ }^{319}$ (iii) plasma power discharge; ${ }^{17} 11$ (iv) monomer nature (methacrylates, alkyl acrylates, etc.); ${ }^{20-22}(v)$ type of substrates used in the polymer deposition (metals, ceramic, synthetic polymers, skin, etc.); ${ }^{10}$ and (vi) geometry of solid substrates (flat surfaces, rods, fibres, textile or fabrics, etc.). ${ }^{23}$ 24

Considering the abovementioned frameworks, advances have been done with the graft polymerization of thermoresponsive hydrogels to complex geometries. Boscher and co-workers. ${ }^{25}$ reported the use of atmospheric pressure plasma-initiated chemical vapor deposition (APPiCVD) process to study the copolymerization of $N$-vinyl caprolactam (NVCL) with ethylene glycol dimethacrylate (EGDMA), in stainless steel plain substrates. They succeeded in obtaining nanometric layer of thermoresponsive polymer, by using ultrashort plasma pulses, without any solvents or chemical initiators. The same atmospheric plasma equipment was used to coat cellulose fabrics with poly(diethylallylphosphate) molecules, ${ }^{21}$ as well as polyacrylates on silicon wafers ${ }^{22}$. The mechanisms of graft copolymerizations, under CVD process, were also elucidated with the help of infrared (FTIR), X-ray photoelectron spectroscopy (XPS) and high-resolution mass spectrometry (HR-MS) analyses. 
Among the large variety of stimuli-responsive soft materials, NIPAAm polymer (PNIPAAm)-based hydrogels, with triggerable phase transition behaviour, ${ }^{8,26}$ represent the quintessence of thermoresponsive materials, displaying adaptive changes in mechanical properties related to the amount of water expelled or absorbed from the hydrogel network. ${ }^{8,27}$ However, AP-PiCVD technique of solid NIPAAm monomer has some drawback due to its low vapor pressure $\left(2.32 \times 10^{-2} \mathrm{~mm} \cdot \mathrm{Hg}\right.$ at $\left.25^{\circ} \mathrm{C}\right)$. By contrary, when the monomers are dissolved in aqueous solutions, atmospheric pressure dielectric barrier discharge (DBD) can be applied to obtain PNIPAAm polymer and copolymers, adhered to glass substrate, with moderate plasma power (10-30 W) and plasma exposure time (10-15 min), as previously reported by Molina et. al. ${ }^{14,28}$

The present work represents an initial study for the future development of a new generation of surgical meshes, able to interact with the complex three-dimensional structure of biological tissues, as smart material working as self-evolving motion sensor. The idea is to create a bilayer mesh, where one component is the polypropylene (PP), as hard material; and the other is the thermoresponsive hydrogel (PNIPAAm), as soft unit. The PP-coated PNIPAAm system is expected to be able to swell and shrink in response to temperature changes. Therefore, here we describe a facile approach for the obtaining of a new generation of thermoresponsive surgical meshes, made of iPP fibres and PNIPAAm covalently bonded. First, the functionalization of iPP surface, composed by monofilament fibres, was investigated under controlled oxygenplasma activation $\left(\mathrm{O}_{2}\right.$-plasma). Further, the graft copolymerization of $\mathrm{N}$-isopropylacrylamide (NIPAAm) and $N, N$-methylene bis(acrylamide) (MBA) monomers onto iPP porous architecture was studied by chemical radical polymerization. Moreover, the mechanism of covalent bonding between fibres and polymer, as well as the PNIPAAm growth were discussed.

As a result, the PP-PNIPAAm bilayer mesh obtained by this approach are expected to endow the non-absorbable patch repair with new properties, independent of those of the bulk polymer, for future applications as thermosensitive biomedical sensor.

\section{Experimental section}

\subsection{Materials}

Monofilament Optilene ${ }^{\circledR}$ Mesh LP was used as substrate for plasma treatment and was kindly supplied by B Braun Surgical, S.A. (Rubí, Barcelona, Spain). Optilene ${ }^{\circledR}$ Mesh LP is a lightweight ( $36 \mathrm{~g} / \mathrm{cm}^{2}$ of weight per area), flexible and non-absorbable iPP mesh, with $1.0 \pm 10 \%$ $\mathrm{mm}$ pore size and $0.39 \pm 10 \% \mathrm{~mm}$ of thickness. Other reagents used were: NIPAAm (purity 99\%, CAS 2210-25-5), MBA (Reagent Plus 99\%, CAS 110-26-9), $N, N, N^{\prime}, N^{\prime}$ - tetramethylethylenediamine (TEMED, Reagent Plus 99\%, CAS110-18-9), all supplied by Sigma-Aldrich Spain; and ammonium persulfate (APS, purity $98 \%$ CAS7727-54-0), provided by Panreac S.A. All reagents were used as received. Milli-Q water grade $(0.055 \mathrm{~S} / \mathrm{cm})$ was used in all synthetic processes. Nitrogen used was pure grade ( $99.995 \%$ purity).

\subsection{Oxygen plasma treatment}

The graft polymerization of NIPAAm on the iPP mesh was carried out in two successive steps. The first step was the plasma irradiation of the mesh surface to generate radical species onto its surface; while the second step was the grafting and polymerization of NIPAAm and MBA monomers, by using radical polymerization. The first step, the mesh surface was exposed to plasma, using two different plasma power discharges ( $250 \mathrm{~W}$ and $500 \mathrm{~W}$ ) and different reactor pressures before oxygen-plasma injection, to obtain polymer radicals on its surface, while the second step was the grafting and polymerization of NIPAAm monomer by using radical polymerization.

The plasma activation of iPP fibres was carried out with low-pressure radio-frequency (RF) plasma ( $80 \mathrm{MHz}$ ), by using a LFG generator 1000 W (Diener Electronic GmbH Co., Germany) and a chamber of $25 \mathrm{dm}^{3}$. In this equipment, the oxygen plasma is created by the RF discharge using low pressure when the gas is passed. The general conditions used for the chamber are: low vacuum pressure of $0.20,0.10$ and 0.07 mbar (depending on the power plasma setup) and a gas flow fixed in 180 seconds and $20 \mathrm{sccm}$ (standard cubic centimetre). To sum up, the samples $\left(100 \mathrm{~cm}^{2}\right)$ were placed within the chamber and the whole system was purged until the desired vacuum pressure and filled with oxygen gas. After the plasma treatment, all samples were stored under vacuum for a few days, if not used immediately. The samples were coded as "untreated" and "treated by plasma" to identify them before and after plasma irradiation, respectively.

\subsection{Graft copolymerization of NIPAAm and MBA onto polypropylene mesh}

Graft copolymerization of NIPAAm monomer onto the iPP meshes treated by $\mathrm{O}_{2}$-plasma was carried out adapting a procedure described in the literature. ${ }^{29}$ Briefly, NIPAAm monomer $(0.5658 \mathrm{~g}, 250 \mathrm{mM})$, MBA cross-linker $(0.0031 \mathrm{~g}, 1 \mathrm{mM})$ and TEMED catalyst $(0.0065 \mathrm{~g}, 2.77 \mathrm{mM})$ were dissolved in $20 \mathrm{~mL}$ of water in a reaction vessel. After total dissolution, all reagents were mixed with a pre-weighed Optilene ${ }^{\bullet}$ mesh LP $\left(4 \mathrm{~cm}^{2}\right)$ in the same reaction vessel. The solution was stirred and nitrogen gas flow was bubbled through for 30 minutes to remove dissolved oxygen, before the addition of the initiator. Then, $0.15 \mathrm{~mL}$ of APS $(370 \mathrm{mM})$ aqueous solution was added to the vessel to initiate the polymerization. The temperature was maintained at $30^{\circ} \mathrm{C}$ with water bath. After 20 hours of reaction, the samples, composed by Optilene mesh LP and PNIPAAm, were extracted and poured onto $400 \mathrm{~mL}$ of deionized water, stirring for 4 hours for purification. All iPP- $g$-PNIPAAm samples were dried at $30^{\circ} \mathrm{C}$ overnight under vacuum.

\subsection{Physical-chemical characterization}


X-Ray photoelectron spectroscopy (XPS) analyses were used for the detection of chemical species formed on the fibre surface, supported on gold substrates, before and after the oxygen plasma exposure. The assays were performed on a SPECS system equipped with an Al anode XR50 source operating at $150 \mathrm{~mW}$ and a Phoibos MCD-9 detector. The pressure in the analysis chamber was always below $10^{-7}$ Pa. The pass energy of the hemispherical analyzer was set at $25 \mathrm{eV}$ and the energy step was set at $0.1 \mathrm{eV}$. Data processing was performed with the Casa XPS program (Casa Software Ltd., UK). The atomic concentration has been calculated subtracting the values coming from eventual contamination of the gold substrate employed during the XPS analysis.

Raman spectra were acquired using a Renishaw dispersive Raman microscope spectrometer (model InVia Qontor, GmbH, Germany) and a Renishaw WiRE software. The spectrometer is equipped with a Leica DM2700 M optical microscope, a thermoelectrically cooled chargecoupled device $(C C D)$ detector $\left(-70{ }^{\circ} \mathrm{C}, 1024 \times 256\right.$ pixels) and a spectrograph scattered light with a 2400 lines $\mathrm{mm}^{-1}$ of grating. The experiments were performed with a $532 \mathrm{~nm}$ excitation wavelength and with a nominal laser power between 1 and $100 \mathrm{~mW}$ output power. The exposure time was $10 \mathrm{~s}$, the laser power was adjusted to $1 \%$ of its nominal output power and each spectrum was collected with 3 accumulations. All Raman spectra were collected in a spectral range from 600 to $4000 \mathrm{~cm}^{-1}$ with the same measurement parameters. Raman spectroscopy was used to identify the main absorption bands, and to estimate the crystallinity and the degree of isotacticity of iPP matrix, ${ }^{30-}$ 32 before and after plasma functionalization (details included in the ESI).

\subsection{Surface topography}

Scanning electron microscopy (SEM) were carried out using a Focused Ion Beam Zeiss Neon40 scanning electron microscope equipped with an energy dispersive X-ray analysis (EDX) spectroscopy system and operating at $5 \mathrm{kV}$. The SEM was used to examine the surface morphology of pristine iPP fibres and iPP fibres covered by PNIPAAm hydrogel (iPP-g-PNIPAAm). The meshes were mounted on a double-side adhesive carbon disc and sputter-coated with a thin layer of carbon to prevent sample charging problems. Worth mentioning, in order to better observe the internal microstructure of the porous gel, in this step, the samples were dried by freeze drying (after the graft reaction) and the cross-section of mesh fibres were observed under cryo-fracture.

Atomic force microscopy (AFM) images were taken with a Molecular Imaging PicoSPM and a NanoScope IV controller, under ambient conditions. The AFM tapping mode was operated at constant deflection. The row scanning frequency was set to $1 \mathrm{~Hz}$. AFM measurements were performed on various parts of the fibres, previously to the hydrogel deposition, which provided reproducible images. The scan window sizes used were $10 \times 10 \mu \mathrm{m}^{2}$. The statistical application of the NanoScope Analysis software was used to determine the root mean square roughness $\left(R_{q}\right)$, which is the average height deviation taken from the mean data plane. Additional AFM images were taken with low polymerization time (1h), to corroborate with mechanism of adhesion and grafting among fibres and PNIPAAm-co-MBA copolymer.

\subsection{Volume phase transition temperature (VPTT) of the bilayer system}

The volume phase transition temperature (VPTT) and the thermoresponsiveness of the grafted hydrogel onto iPP surface have been evaluated by optical microscopy with increase temperature. A Zeiss Axioscop 40 Pollight polarizing microscope (CarlZeiss, Göttingen, Germany) was employed to measure the length of PNIPAAm gel (captured by micrographs and calculated as the distance of PNIPAAm side chains from iPP fiber where the gel is grafted), while temperature was controlled with a Linkam system having a THMS 600 heating and freezing stage connected to an LNP94 liquid nitrogen cooling system (LinkamScientific, Tadworth,UK). A Zeiss Axios Cam MRC5 digital camera (Carl Zeiss, Göttingen, Germany) was employed to get micrographs at appropriate temperature intervals. The variation of the gel length was evaluated according to the equation (1):

$$
\text { Variation of hydrogel length }(\%)=\frac{\mathrm{L}_{\mathrm{f}}}{\mathrm{L}_{0}} \times 100
$$

where $L_{f}$ and $L_{0}$ are the difference of increment on lengths $(\mu \mathrm{m})$ of the PNIPAAm gel, well adhered to the iPP mesh, under specific temperature and respect to the final and initial states, respectively.

The graft yield (GY) and the equilibrium swelling ratio (ESR) of crosslinked PNIPAAm gel grafted onto the surface of the low-density iPP meshes were also evaluated. The procedure is reported in the ESI.

\section{Results and discussion}

\subsection{Process of prosthesis functionalization and mechanism of PNIPAAm-co-MBA chain growth}

The main steps performed in the present study are summarized as follow: (A) iPP mesh cleaning with ultrasound bath and dry; (B) iPP-mesh plasma exposure by using different conditions of $\mathrm{O}_{2}$ pressure and plasma power; and $(C)$ graft copolymerization of NIPAAm onto iPP fibres and among polymer chains, with constant crosslinker ratio and polymerization time. The mechanism overview of prosthesis functionalization and PNIPAAm deposition is schematized in the Figure 1. 
This section sketches the main reactions involved in those processes, based on the analytical results carried out in the present study. More in detail, the general mechanism proposed for the hydrogel deposition to iPP fibres (Figure 2 ) is similar to that reported in the literature for the grafting of PNIPAAm onto cellulose nanocrystals ${ }^{33}$. The major novelty of our approach relies on the need of create high density of reactive functional groups in the polypropylene fibres by applying controlled plasma discharge. Therefore, the mechanism consists of three main steps. After the functionalization of the mesh by plasma ( $1^{\text {st }}$ step), an initiation reaction by the sulphate anion-radical ( $2^{\text {nd }}$ step), induced by the decomposition of APS and kinetically accelerated by the presence of the catalyst (TEMED) takes place, which abstracts hydrogen from one of the functional groups in the side chains (i.e., $\mathrm{COOH}, \mathrm{C}-\mathrm{OH}, \ldots$ ) of the

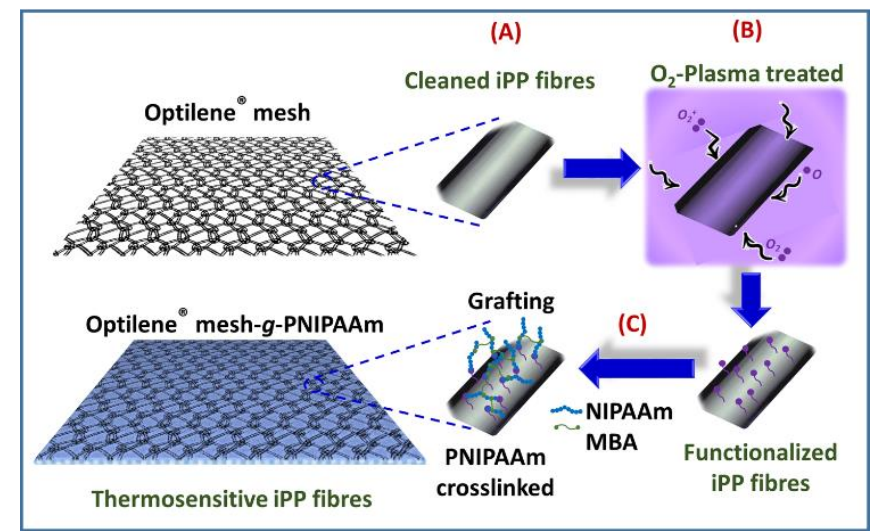

Figure 1. Polypropylene mesh submitted to plasma exposure and PNIPAAm deposition: (A) cleaning process, (B) plasma exposure, and (C) graft copolymerization of NIPAAm and MBA monomers.

substrate to form the corresponding radical. ${ }^{34}$ Then the resulting macroradicals initiate the graft copolymerization of NIPAAm leading to the appearance of the graft copolymer, called iPP-g-PNIPAAm ( $3^{\text {rd }}$ step). Since the crosslinking agent (MBA) is present in the reaction mixture, the growth of the hydrogel among fibres and between NIPAAm chains, is ensured by the effect of this co-monomer.

For the free radical mechanism, the hydrogen abstraction from carboxylic acid is the key step, even at neutral $\mathrm{pH} .{ }^{35}$ This group is present in its deprotonated state. Thus, the $\mathrm{C}-\mathrm{OH}$ functional groups are expected to be the more active species, interacting with the sulphate anionradical to create the macroradical. After the formation of $\mathrm{HSO}_{4}{ }^{-}$species ( $4^{\text {th }}$ step), the $\mathrm{pH}$ of the solution diminishes due to the $\mathrm{H}_{3} \mathrm{O}^{+}$formation (from $\mathrm{HSO}_{4}^{-}$equilibrium in water, $4^{\text {th }}$ step). In this case the carboxylic group could be protonated and could also be active for further grafting reactions. Furthermore, the presence of high concentrations of $\mathrm{C}-\mathrm{OH}$ and carboxylic groups were meticulously investigated by XPS (section 3.2), by Raman and FTIR spectra (section 3.3). The results will be discussed in the following sections.

Furthermore, the covalent bonding between NIPAAm monomer and MBA crosslinker to the fibre surface was observed by AFM, with low reaction polymerization time (section 3.5). The hydrogel growth starts above the fibre surface and continues among PNIPAAm network. The resulting thermoresponsive iPP mesh system, after $20 \mathrm{~h}$ of reaction, is able to respond to temperature changes close to the volume phase transition temperature (VPTT) of PNIPAAm molecules (section 3.6). 


\section{Postprint}

$\underline{1^{\text {st }} \text { step: Surface functionalization by } \mathrm{O}_{2} \text {-plasma treatment }}$

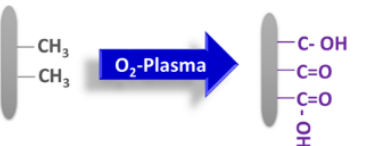

Surface oxidation

$2^{\text {nd }}$ step: radical polymerization of NIPAAm-co-MBA under APS activation

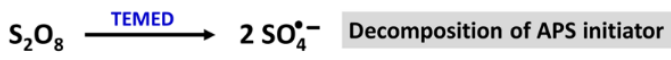

$3^{\text {rd }}$ step: Grafting of NIPAAm onto plasma treated Optilene ${ }^{\circ}$ mesh

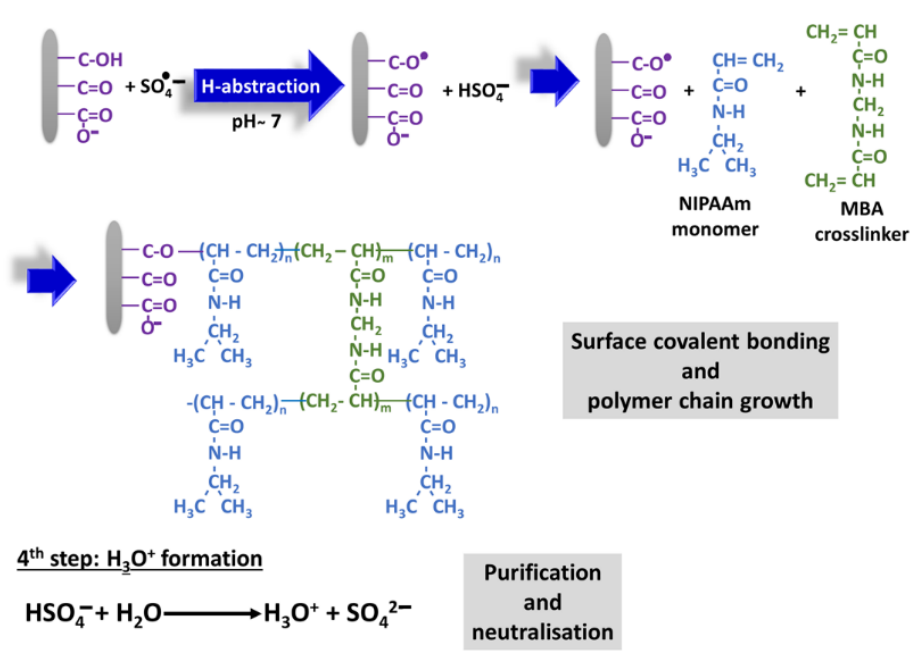

Figure 2. Schematic illustration of the reactions mechanism involved in the main steps for the thermoresponsive PNIPAAm hydrogel deposition in iPP fibres, promoted by the exposure of its surface to plasma discharge.

The plasma functionalization of Optilene ${ }^{\circledR}$ meshes LP was carried out by changing the values of the purging pressure inside the reactor chamber and the plasma power discharge, i.e. by modifying these two parameters of the plasma reactor. The influence of such parameters on the functionalization and further hydrogel adhesion will be discussed in the next section.

\subsection{Effect of the purging pressure and plasma power in the surface functionalization of polypropylene mesh}

More specifically, the purging pressure is the final pressure that the equipment reaches in the vacuum step before the injection of oxygen and plasma discharge, affecting the elimination of the air inside the equipment and, therefore, determining the concentration of $\mathrm{C}$ and $\mathrm{O}$ elements upper the meshes surfaces. Consequently, this parameter is crucial for further deposition of thermoresponsive gel by reactive covalent bonding. In this work, we have investigated by XPS and Raman the effect of three different values of purging pressure $(0.20,0.10$ and $0.07 \mathrm{mbar}$ ) on the $\mathrm{O}_{2}$-plasma functionalization of meshes surfaces. Table S1 reports the atomic percentage of $\mathrm{C}$ and $\mathrm{O}$ detected by XPS for all samples investigated, before and after plasma discharge, at different pressure values applying a plasma power of $500 \mathrm{~W}$, a plasma time of $180 \mathrm{~s}$ and a pressure of $\mathrm{O}_{2}$-plasma of $0.30 \mathrm{mbar}$. After lowering the pressure inside chamber, it was observed a decay in the atomic percentage of $C$ that corresponds to an increase of the oxygen from $1.72 \pm 0.57 \%$, in the untreated sample, to $20.99 \pm 0.14 \%, 24.38 \pm 1.58$ $\%$ and $25.54 \pm 2.35 \%$ for samples activated by plasma at $0.20,0.10$ and 0.07 mbar, respectively. The atomic concentrations of $C$ and $O$ reported in Table S1 confirm that the $\mathrm{O}_{2}$ /air ratio increases with decreasing the pressure inside the chamber. It is worth noting that investigations in this parameter are usually skipped in such kind of plasma treatment. The knowledge about the influence of the purging down pressure is expected to help the optimization of the cost related to the vacuum step, that is one of the negative aspect of the low pressure vacuum processes. ${ }^{18}$

The XPS survey spectra of pristine iPP meshes and samples irradiated by $\mathrm{O}_{2}$-plasma, by keeping the pressure and plasma discharge constant $(0.20$ mbar and $500 \mathrm{~W}$, respectively), is showed in the Figure 3a. A shift to higher binding energy and an increase of the intensity of 
peaks at 287.2 and $533.1 \mathrm{eV}$ ( $\mathrm{C}$ 1s and $\mathrm{O}$ 1s, respectively), were observed. Peaks related to gold ( $\mathrm{Au}$ ), which belong to the holder employed to support the samples during the measurements, and were also reported elsewhere. ${ }^{36}$ The relative deconvolution of $\mathrm{C} 1 \mathrm{~s}$ and $\mathrm{O} 1 \mathrm{~s}$ peaks revealed the formation of highly oxidized carbon such as carboxylate and carbonate groups, as displayed in the high resolution XPS spectra (Figures $3 \mathrm{~b}-\mathrm{e}$ ). As it was expected, the analysis of the $\mathrm{C}$ 1s signal on iPP mesh fibres shows the presence of different chemical species after plasma treatment. The pristine Optilene ${ }^{\bullet}$ meshes (Figure $3 \mathrm{~b}$ ) present bands at 284.3 and $285.6 \mathrm{eV}$, which have been attributed to the $\mathrm{C}-\mathrm{C}$ and $\mathrm{C}-\mathrm{H}$ bonds, respectively, ${ }^{37-39}$, whereas the bands at higher binding energies were assigned to $\mathrm{C}-\mathrm{O}$ and $\mathrm{C}=\mathrm{O}$ functional groups (at 286.5 and $288.3 \mathrm{eV}$, respectively), suggesting that the untreated iPP fibres contains low-level of oxidized carbon centers on its surface. ${ }^{40}$ Such binding energies were slight different from that of iPP flat and solid films (Figure S1), before plasma exposure. A deeper discussion was inserted in the ESI to support those results in solid and non-porous surface, compared to the complex architecture of iPP meshes. After plasma discharge (Figure 3c), the amount of $\mathrm{C}-\mathrm{H}$ species in iPP meshes decreases (peak pointed at $285.6 \mathrm{eV}$ ), while the amount of $\mathrm{C}-\mathrm{O}$ and $\mathrm{C}=\mathrm{O}$ increases. Furthermore, new peaks at 289.1 and $291.0 \mathrm{eV}$ appeared and were ascribed to $\mathrm{O}-\mathrm{C}=\mathrm{O}$ groups and $\mathrm{CO}_{3}{ }^{2-}$ species. ${ }^{39} \mathrm{More}$ specifically, Figures 3d-e indicate that the peaks at $532.2 \mathrm{eV}(\mathrm{C}-\mathrm{O})$ and $533.6 \mathrm{eV}(\mathrm{C}=\mathrm{O})$ decreased and increased for the untreated and plasma treated surfaces, respectively, whereas the appearance of a new peak at $535.6 \mathrm{eV}$, due to $\mathrm{O}-\mathrm{C}=\mathrm{O} / \mathrm{CO}_{3}{ }^{2-}$ linkages, has been concomitantly observed only for the meshes under plasma exposure. .2,16,23 $^{2}$
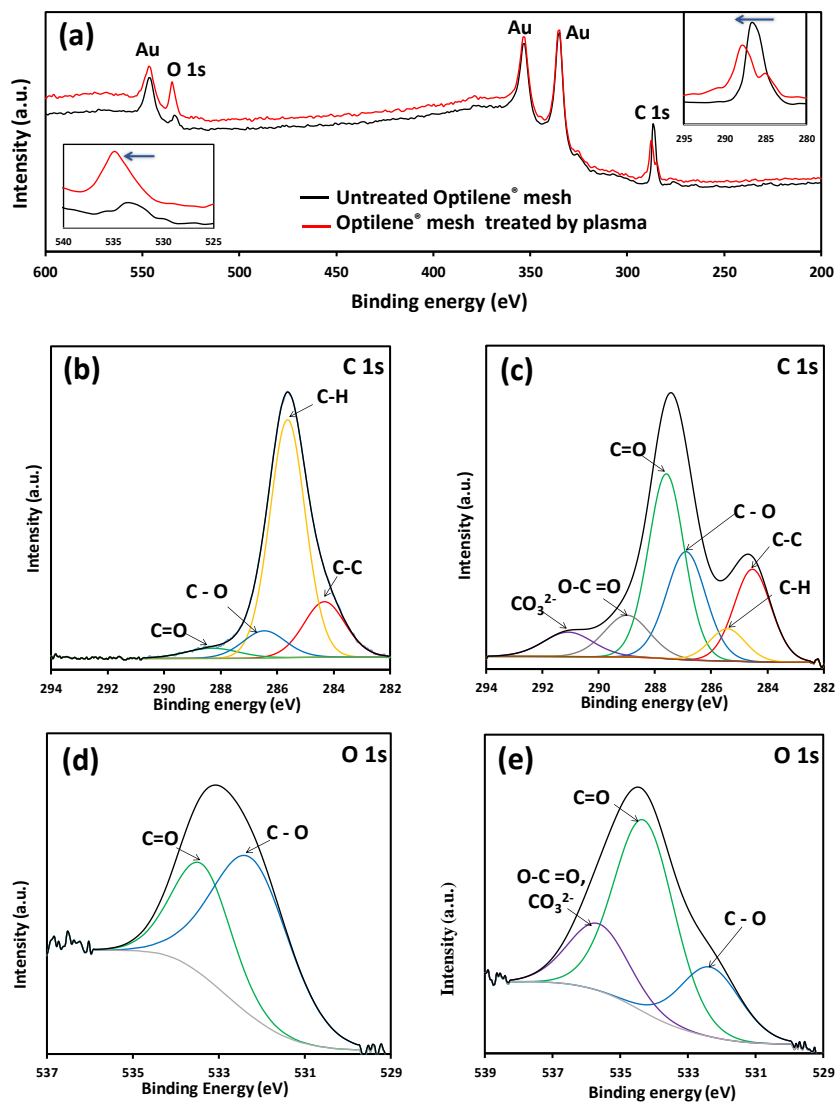

Figure 3. (a) XPS survey spectra of pristine Optilene ${ }^{\circledR}$ mesh and iPP meshes after $\mathrm{O}_{2}$-plasma surface functionalization at constant purging down pressure, power discharge and time ( 0.20 mbar, $500 \mathrm{~W}$ and $180 \mathrm{~s}$, respectively). (b-d) High resolution XPS spectra of $\mathrm{C} 1 \mathrm{~s}$ and $\mathrm{O} 1 \mathrm{~s}$ elements, where (b,d) represents the pristine polymer and (c,e) the iPP mesh after plasma exposure.

Variations of oxygen/carbon $(\mathrm{O} / \mathrm{C})$ ratio, shown in Table S2 outline that oxygen is enhanced after plasma treatment, increasing from $0.017 \pm 0.006$ for pristine Optilene ${ }^{\circledR}$ mesh to $0.266 \pm 0.002,0.323 \pm 0.028$ and $0.344 \pm 0.042$ for Optilene ${ }^{\circledR}$ mesh after plasma treatment at $0.20,0.10$ and 0.07 mbar as purging pressure, respectively. Such high $\mathrm{O} / \mathrm{C}$ contents could be correlated to the high plasma power employed $(500 \mathrm{~W})$, as previously reported by other authors working at similar values of plasma power. An O/C ratio of 0.394 was found applying a plasma power of $400 \mathrm{~W}$ by Kravets et al. ${ }^{41}$; whereas 0.45 was obtained at $600 \mathrm{~W}$ in the study developed by Tyan et al. ${ }^{42}$.

Taking into account the previous results, the effect of the plasma power in the iPP fibres was evaluated by decreasing the power of a factor of twice ( $250 \mathrm{~W})$, and maintaining the purging pressure inside reactor at 0.07 mbar. Comparing the lowest plasma discharge (250 W) with the highest value $(500 \mathrm{~W}$ ), XPS results evidenced a decrease in the C 1 s content (from $79.93 \pm 1.75 \%$ to $74.46 \pm 2.35 \%$ ) with increasing power irradiation, whereas the amount of $\mathrm{O} 1 \mathrm{~s}$ increases (from $20.07 \pm 1.75 \%$ to $25.54 \pm 2.35 \%$, Table 1 ). Thus, the plasma power has a reduced influence on the amount of active groups in the oxidized surface. This fact leads to the conclusion that the key parameter to be 
controlled is the ratio of $\mathrm{O}_{2}$ /air, imposed by the plasma reactor when maintaining the power constant and varying the purging down pressure of air inside the chamber.

Table 1. Atomic concentrations of C1s and $\mathrm{O} 1 \mathrm{~s}$ obtained by XPS high resolution spectra, discriminated by elements and functional groups.

\begin{tabular}{|c|c|c|c|c|c|c|c|c|}
\hline \multirow[t]{11}{*}{ Sample code } & $\begin{array}{c}\text { Plasma parameters } \\
\text { (Pressure, Plasma power) }\end{array}$ & Element & $\begin{array}{l}\text { Atomic conc. } \\
(\%)\end{array}$ & $\mathrm{O} / \mathrm{C}$ ratio & $\begin{array}{l}\text { Functional } \\
\text { groups }\end{array}$ & $\begin{array}{c}\text { Binding } \\
\text { energy }(e V)\end{array}$ & $\begin{array}{l}\text { Atomic conc. } \\
(\%)\end{array}$ & $\begin{array}{c}\mathrm{C}-\mathrm{O} \backslash \mathrm{O}-\mathrm{C}=\mathrm{O} \\
\text { ratio }\end{array}$ \\
\hline & & C (1s) & $98.28 \pm 0.57$ & $0.017 \pm 0.006$ & C 1s (C-O) & 286.5 & $9.6 \pm 0.36$ & - \\
\hline & & $\mathrm{O}(1 \mathrm{~s})$ & $1.72 \pm 0.57$ & & $\mathrm{C} 1 \mathrm{~s}(\mathrm{C}=\mathrm{O})$ & 288.3 & $4.1 \pm 0.25$ & - \\
\hline & & & & & $\mathrm{C} 1 \mathrm{~s}(\mathrm{C}-\mathrm{O})$ & 286.9 & $24.2 \pm 0.13$ & \\
\hline & & $C(1 s)$ & $74.46 \pm 2.35$ & $0.344 \pm 0.042$ & $\mathrm{C}$ 1s $(\mathrm{C}=\mathrm{O})$ & 287.6 & $33.3 \pm 0.09$ & $1.63 \pm 0.03$ \\
\hline & & $\mathrm{O}(1 \mathrm{~s})$ & $25.54 \pm 2.35$ & & $\mathrm{C} 1 \mathrm{~s}(\mathrm{O}-\mathrm{C}=\mathrm{O})$ & 289.1 & $14.9 \pm 0.35$ & \\
\hline & & & & & $\mathrm{C} 1 \mathrm{~s}\left(\mathrm{CO}_{3}{ }^{2-}\right)$ & 291.2 & $7.3 \pm 0.40$ & \\
\hline & & & & & C 1s (C-O) & 286.9 & $27.43 \pm 0.61$ & \\
\hline & & $C(1 s)$ & $79.93 \pm 1.75$ & $0.251 \pm 0.028$ & $\mathrm{C}$ 1s $(\mathrm{C}=\mathrm{O})$ & 287.6 & $26.68 \pm 1.80$ & $3.41 \pm 0.11$ \\
\hline & & $\mathrm{O}(1 \mathrm{~s})$ & $20.07 \pm 1.75$ & & $\mathrm{C}$ 1s $(\mathrm{O}-\mathrm{C}=\mathrm{O})$ & 289.1 & $8.04 \pm 0.09$ & \\
\hline & & & & & $\mathrm{C} 1 \mathrm{~s}\left(\mathrm{CO}_{3}{ }^{2-}\right)$ & 290.9 & $10.5 \pm 2.27$ & \\
\hline
\end{tabular}
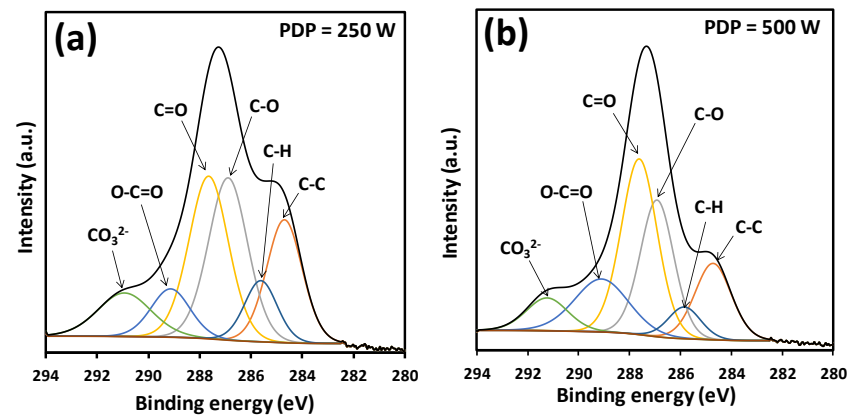

Figure 4. Comparison of $\mathrm{C} 1 \mathrm{~s}$ high resolution spectra of iPP mesh irradiated with plasma power of (a) $250 \mathrm{~W}$ and (b) $500 \mathrm{~W}$. The purging pressure and the time of plasma exposure were maintained constant $(0.07 \mathrm{mbar}$ and $180 \mathrm{~s}$, respectively).

The areas and the position of the deconvolution analysis of $\mathrm{C} 1 \mathrm{~s}$ are reported in Figures $4 \mathrm{a}-\mathrm{b}$ and Table 1 . The peak at about $287 \mathrm{eV}$ has been assigned to $\mathrm{C}-\mathrm{O}$ single bonds (i.e. $\mathrm{C}-\mathrm{OH}$ or $\mathrm{C}-\mathrm{O}-\mathrm{C}$ groups). The other two peaks, which exhibits higher binding energies have been attributed to the most oxidized carbon species, such as the $\mathrm{C}=\mathrm{O}$ and $\mathrm{O}-\mathrm{C}=\mathrm{O}$ groups at about $288-289 \mathrm{eV}$ and the $\mathrm{CO}_{3}{ }^{2-}$ group at $290-291 \mathrm{eV}$. The polar groups observed for the Optilene ${ }^{\circledR}$ mesh LP without plasma activation, which mostly involved $\mathrm{C}-\mathrm{O}$ and $\mathrm{C}=\mathrm{O}$ bonds (reported in the Table S1), corresponded to a total oxygen concentration of only $1.72 \pm 0.57 \%$. Figure $4 \mathrm{~b}$ shows a slight increment of the $\mathrm{C}=\mathrm{O}$ peak when the highest plasma discharge is used $(500 \mathrm{~W})$.

Table 1 shows the ratio between oxygen and carbon $(\mathrm{O} / \mathrm{C})$ calculated by atomic concentration of $\mathrm{C} 1 \mathrm{~s}$ and $\mathrm{O}$ 1s and between $\mathrm{C}-\mathrm{O}$ and $\mathrm{O}-\mathrm{C}=\mathrm{O}$ functional groups derived from the deconvolution of the $\mathrm{C} 1 \mathrm{~s}$ XPS peak. Regardless of the atomic concentration of oxidized functional groups, the subtle augment of $\mathrm{C}-\mathrm{O} / \mathrm{O}-\mathrm{C}=\mathrm{O}$ ratio at $500 \mathrm{~W}$ could be attributed to the high concentration of $\mathrm{C}-\mathrm{OH}$ groups (very active for the grafting of NIPAAm molecules). Therefore, additional experiments of molecular spectroscopy, to corroborate the nature of C-O species above iPP fibres, were necessary and will be discussed in the next section. Overall, these results allow us to conclude that complex 2D structures, like PP meshes, can be oxidized in a similar manner than flat surfaces, if the plasma operational parameters are controlled. Our results are fully consistent with some previous studies in which the time of plasma exposure was varied but the plasma discharge was kept fixed. ${ }^{12,16,23}$

\subsection{Effect of the plasma power in the molecular structure of polypropylene meshes}

Plasma-introduced functional groups that were additionally identified and qualified by FTIR and Raman spectroscopies. Therefore, deconvolution of the broad adsorption peaks being related to the $\mathrm{O}-\mathrm{H}$ vibration region $\left(3000-4000 \mathrm{~cm}^{-1}\right.$, Figures $\left.5 \mathrm{a}-\mathrm{c}\right)$, revealed the presence of new absorption peaks at about 3587 and $3735 \mathrm{~cm}^{-1}$, when $250 \mathrm{~W}$ of plasma discharge was applied.12 Besides as well as shifting of the peak situated at about $3660 \mathrm{~cm}^{-1}$ towards higher wavenumbers $\left(3678 \mathrm{~cm}^{-1}\right)$ was observed. These findings may imply on an induced hydrophilicity of the PP mesh surface after plasma treatment and a higher amount of hydroxyl groups suitable for new grafting reactions. On the other hand, deconvolution of carbonyl vibration region (Figures $5 b-d$ ) demonstrates the existence of different nature of carbonyl groups, which 
are: $1652 \mathrm{~cm}^{-1}$ (asymmetrical stretching vibration of deprotonated carboxyl group vibrations), $1702 \mathrm{~cm}^{-1}$ (protonated C=0), $1750 \mathrm{~cm}^{-1}$ (aldehyde) and $1780 \mathrm{~cm}^{-1}$ (ketone). As shown in the Figure $5 \mathrm{~d}$, carbonyl vibration band decreased in the PP mesh treated with plasma power of $250 \mathrm{~W}$.

Moreover, the ratio between the areas of peaks related to the $\mathrm{O}-\mathrm{H}$ vibration region $\left(\mathrm{A}_{\mathrm{O}-\mathrm{H}}\right)$ and the peak corresponding to the asymmetrical stretching of carboxyl group $\left(1652 \mathrm{~cm}^{-1}\right)\left(\mathrm{A}_{\mathrm{O}-\mathrm{C}=\mathrm{O}}\right)$ was calculated. For both samples $(250 \mathrm{~W}$ and $500 \mathrm{~W})$, the relative concentration of $\mathrm{C}-\mathrm{OH}$ groups is twice for the sample treated at lower plasma discharge than at $500 \mathrm{~W}$ (123.0 and 66.1, $\mathrm{A}_{\mathrm{O}-\mathrm{H}}$ / $\mathrm{A}_{\mathrm{O}-\mathrm{C}=\mathrm{O}}$, respectively); and confirming the results obtained by XPS, reported previously in the Table 1.
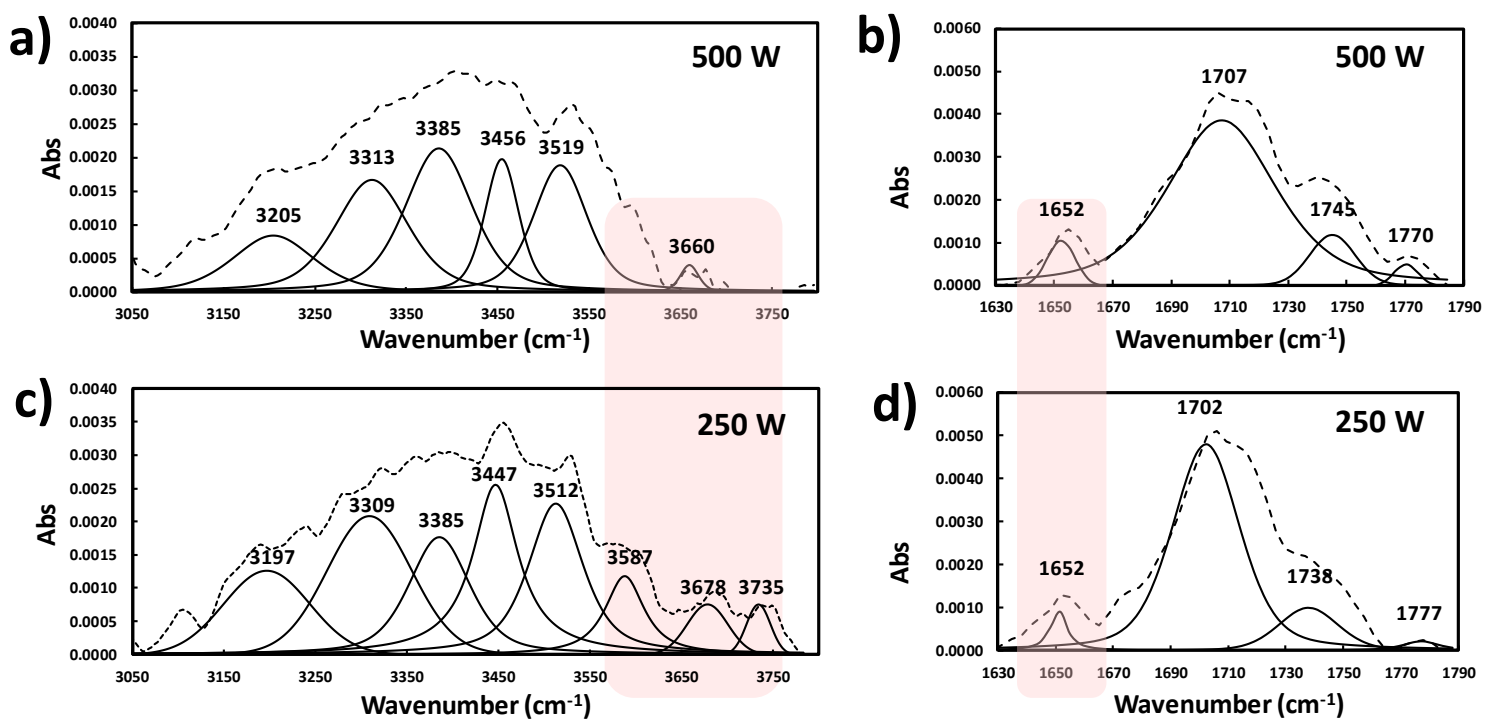

Figure 5. FTIR-ATR spectra of iPP surface after plasma activation at power discharge of $500 \mathrm{~W}$ and $250 \mathrm{~W}$. (a,c) Deconvolution of $3050-3750 \mathrm{~cm}^{-1}$ region (O-H stretching absorption bands), and (b,d) Deconvolution of $1630-1790 \mathrm{~cm}^{-1}$ region ( $\mathrm{C}=\mathrm{O}$ absorption bands). Dashed lines correspond to the experimental spectra, whereas continuous lines represent the deconvolution of the curves. The highlighted absorption bands represent the main changes for the $\mathrm{O}-\mathrm{H}$ stretching vibrations (a and $\mathrm{C}$ ), as well as for $\mathrm{C}=\mathrm{O}$ stretching vibrations.
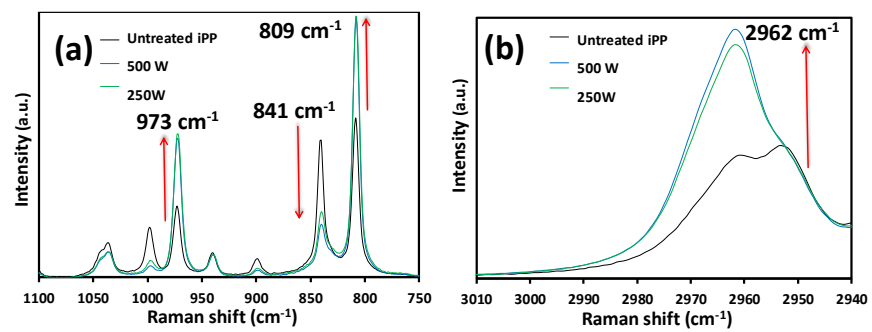

Figure 6. Raman spectra for a shift range of (a) $1100-750 \mathrm{~cm}^{-1}$ and (d) $3010-2940 \mathrm{~cm}^{-1}$, comparing the changes on the chemical structure of iPP fibres after $500 \mathrm{~W}$ and $250 \mathrm{~W}$ of plasma discharge. The time of plasma activation and purging pressure were kept at $180 \mathrm{~s}$ and 0.07 mbar, respectively.

Although the main boundary linkages related to the partial oxidation of the iPP surface were completely elucidated with XPS spectroscopy, the bidimensional architecture of the monofilament mesh was carefully evaluated by micro-Raman spectroscopy.

Raman spectra reported in Figure 6a indicates that the plasma enhances the intensity of the peaks corresponding to the crystalline regions ( 809 and $973 \mathrm{~cm}^{-1}$ ), independently of the plasma discharge. Additionally, the absorption band at $2962 \mathrm{~cm}^{-1} \mathrm{changes}$ its shape with respect to the pristine polymer (Figure $6 \mathrm{~b}$ ). ${ }^{32,43,44}$ This band corresponds to the $\mathrm{C}-\mathrm{H}$ asymmetric stretching vibrations from methyl groups. ${ }^{45}$ Additionally, the reduction of the peak pointed at $841 \mathrm{~cm}^{-1}$ (Figure 6a), originated by the $\mathrm{CH}_{3}$ rocking from amorphous region; and the augment of the peak at $2962 \mathrm{~cm}^{-1}$ (Figure 6b) suggest that the lateral methyl group is slightly modified by the process. Thus, becoming the favourite site for the formation of the oxidized groups observed by XPS analyses and, therefore, the most suitable for the further deposition of the PNIPAAm hydrogel.

The crystallinity of iPP fibres, obtained from equations (1) and (2) (ESI), changed from the initial value of about $43.7 \pm 0.15 \%$ for pristine iPP meshes, to $58.2 \pm 0.58 \%, 71.1 \pm 0.31 \%$ and $67.3 \pm 0.08 \%$ for purging pressure values of 0.20 to 0.10 and 0.07 mbar, respectively, at the 
same plasma discharge (Table S3). By contrary, the intensity of the plasma discharge does not seem to affect in a relevant manner either the crystallinity or the isotacticity of PP fibres. A detailed discussion is supplied in the ESI.

Those changes on the polymer molecular structure are superficial and, therefore, are not expected to affect the mechanical integrity of the fibres. Indeed, additional Raman analysis at different depths were conducted to prove that the effects of the plasma exposure are uniquely affecting the polymer surface (Figures S3-S6, ESI).

\subsection{Surface topography}

In summary, the lowest pressure $(0.07 \mathrm{mbar})$ and the lowest power discharge $(250 \mathrm{~W})$ were taken as the best conditions of plasma exposure for the further chemical deposition of the thermoresponsive PNIPAAm hydrogel on iPP fibres. Both parameters are suitable to create active functional groups onto the inactive surface of the PP meshes, without altering the bulk crystallinity and the isotacticity of the monofilament material respect to the pristine polymer.

AFM and SEM images of the untreated and treated Optilene mesh LP confirmed that iPP fibres are not damaged by the plasma discharge, even if high plasma energy is applied (Figure 7). All samples showed a smooth morphology at the fibre surface with fibrils oriented in the fibre axis direction due to the manufacturing process. The $\mathrm{O}_{2}$-plasma treatment uniquely induces a slight modification in the roughness at nanometric scale, as is shown in the 2D-height, 2D-amplitude and 3D-topographic AFM images of the Optilene mesh LP fibres (Figures 7bd). Thus, the initial surface roughness, expressed in terms of $R_{q}$, is $32.8 \pm 3.4 \mathrm{~nm}$ for the untreated fibres, decreased to $28.6 \pm 2.8 \mathrm{~nm}$ for mesh treated at $500 \mathrm{~W}$ and increased to $49.4 \pm 5.6 \mathrm{~nm}$ for samples treated at $250 \mathrm{~W}$. The fibril orientation at microscale was maintained after plasma exposure.

(a) SEM
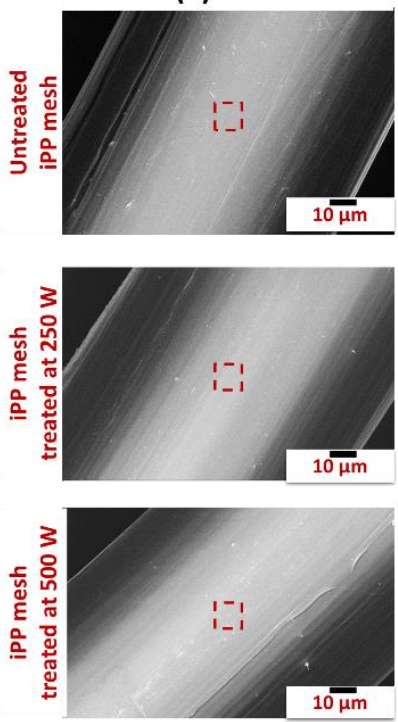

(b) Height
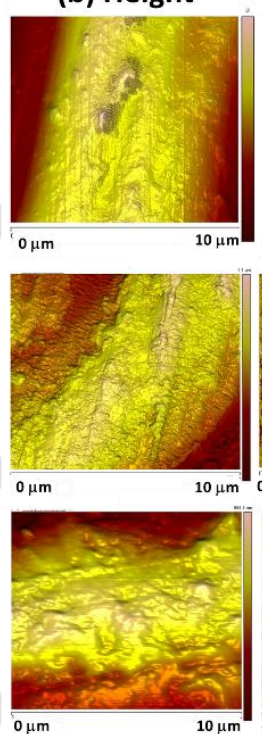

(c) Amplitude
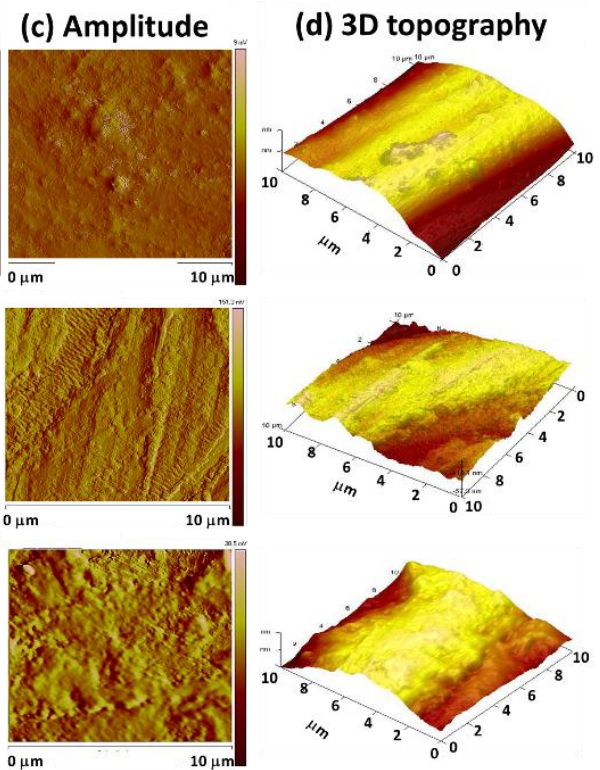

Figure 7. Surface analysis of untreated and plasma treated iPP fibres by using SEM and AFM. (a) High magnification SEM images of iPP mesh fibres (dashed square indicates the zone analysed by AFM); and sets of high resolution topography images (scale:10×10 $\mu \mathrm{m}^{2}$ ). AFM images: (b) height, where dark and brighter areas represent the depressions and the protrusions, respectively, on the 3D surface; (c) amplitude showing the deflection generated by cantilever tip; and (d) 3D topographic image.

\subsection{Effect of plasma functionalization in the immobilization of PNIPAAm molecules onto mesh surfaces}

The plasma reactor conditions had a great influence in the adhesion of PNIPAAm to the 2D-structure of the commercial prosthesis. iPP fibres without oxygen-plasma treatment were not able to maintain the gel adhered to their surfaces due to the lack of polar anchoring groups. Therefore, after gel deposition and purification processes, the PNIPAAm layer is easily removed from the untreated PP fibres.

In order to corroborate the grafting hypothesis supported in the section 3.1, the growth of PNIPAAm-co-MBA molecules was monitored by AFM, at low polymerization time (1h), after plasma treatment. Figure 8 shows that the hydrogel starts to grow from the iPP surface, proving that PNIPAAm chains are not simply crosslinked into a network only in the surroundings of the fibre. At this low polymerization time, it is 
also possible to observe a thin layer of PNIPAAm-co-MBA hydrogel above the iPP fibre, indicating that the grafting starts from the mesh surface.
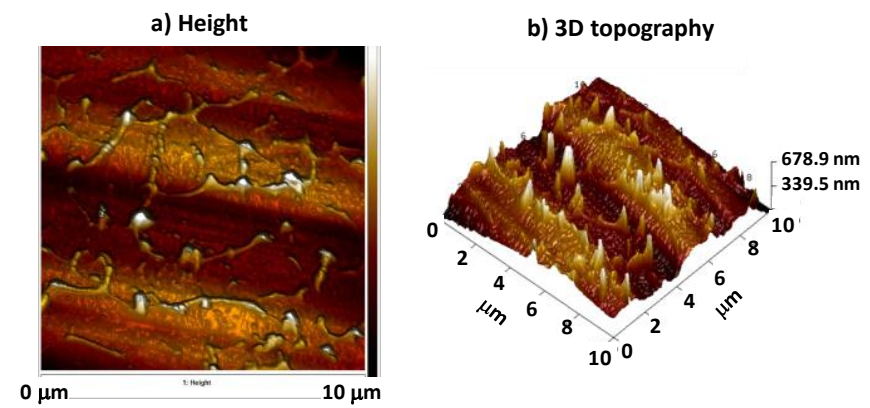

Figure 8. (a) AFM height image and (b) 3D topography of iPP-g-PNIPAAm growth during $1 \mathrm{~h}$ of polymerization reaction over iPP fibre activated at $250 \mathrm{~W}$ of plasma power and 0.07 mbar of purging pressure.

Taking into account the invariable polymerization time and the concentrations of monomer, co-monomer and initiator (section 2.3), the maximum amount of covalent bonds between the PNIPAAm gel and the mesh substrate was obtained with the lowest plasma power (250 W) (Figure 9, Table S4). Thus, the graft yield (eq. 4, ESI) achieved with $250 \mathrm{~W}$ was four times higher $\left(\mathrm{GY}=3.89 \pm 0.12 \mathrm{mg} / \mathrm{cm}^{2}\right)$ than that obtained when the power discharge was $500 \mathrm{~W}\left(\mathrm{GY}=0.93 \pm 0.05 \mathrm{mg} / \mathrm{cm}^{2}\right)$. Good grafting was also obtained when the mesh was prepared with a plasma power of $500 \mathrm{~W}$ and purging pressure of $0.20 \mathrm{mbar}\left(\mathrm{GY}=1.91 \pm 0.18 \mathrm{mg} / \mathrm{cm}^{2}\right)$. Besides, the lowest value of $\mathrm{GY}(0.50 \pm 0.05$ $\mathrm{mg} / \mathrm{cm}^{2}$ ), which was obtained using the pressure of $0.10 \mathrm{mbar}$, corresponds to the lowest oxidized surface density achieved by XPS (Table S1). These results were corroborated by calculating the ratio $A_{0-H} / A_{O-C=O}$ obtained from the deconvolution of FTIR-ATR spectra, as previously described in the section 3.3, for all samples treated with plasma and using the untreated mesh as reference. FTIR-ATR spectra and relative deconvolution for samples treated at $500 \mathrm{~W}$ and 0.2 and $0.1 \mathrm{mbar}$ are reported in the Figure $\mathrm{S} 7$. The $\mathrm{A}_{\mathrm{O}-\mathrm{H}} / \mathrm{A}_{\mathrm{O}-\mathrm{C}=\mathrm{O}}$ ratio was compared to each GY obtained by gravimetric measurements (Figure 9). As can be seen, the GY increases with the $A_{O-H} / A_{O-C=O}$ ratio, confirming that the $C-O H$ functional groups are the active species which interact with the sulphate anion-radical to create the macroradical and initiate the grafting of NIPAAm, as previously discussed in mechanism of polymerization illustrated in the Figure 2.

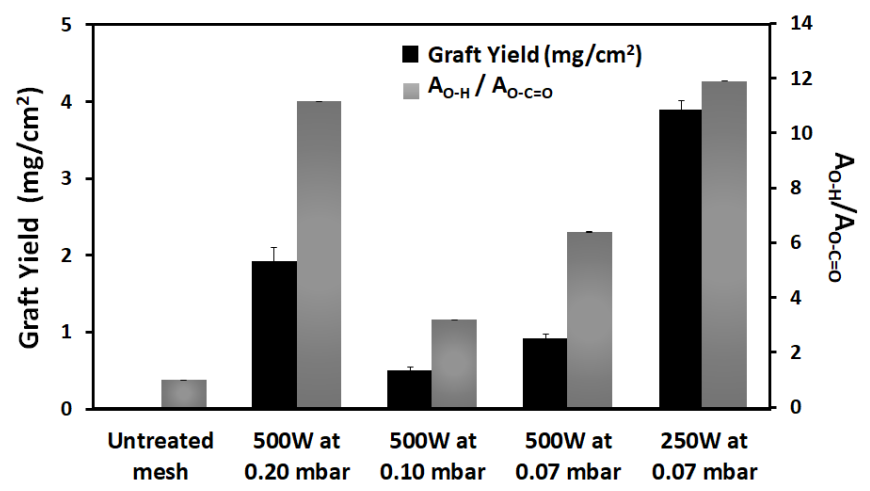

Figure 9. Graft yield and $A_{O-H} / A_{O-C=O}$ ratio from gravimetric measurements and FTIR-ATR spectra deconvolution as a function of plasma power and pressure values.

The equilibrium swelling ratio (ESR, eq. 5, ESI) of all samples was evaluated in a water bath, at 25 o $\mathrm{C}$ for $24 \mathrm{~h}$. In particular, the ESR increased with increasing GY, as observed in the Table S4. Those results have been attributed to the mechanisms of gel linkage and gel growth above the fibres (discussed in the section 3.1), which depends on the type of $\mathrm{C}-\mathrm{O}$ species formed onto the fibres, on the presence of the crosslinking agent (MBA), and on the porosity of film.

Taking into account the surface functionalization optimization and the complete characterization of the hydrogel with FTIR and Raman spectroscopies (Figures S8 and S9, ESI), the lowest and the highest GY films were considered for the deeper study on the mechanism of graft polymerization and hydrogel growth above the bidimensional structure.

\subsection{Thermoresponsive behaviour of PNIPAAm-co-MBA adhered to the iPP mesh fibres}

Although the very low content of PNIPAAm in Optilene ${ }^{\circledR}$ fibres with GY $=0.50 \pm 0.05 \mathrm{mg} / \mathrm{cm}^{2}$ was hardly detected by Raman analysis and optical microscopy (Figure S9a), it was clearly observed by SEM microscopy. The horizontal growth of iPP-g-PNIPAAm gel was detected by 
the sequence of SEM micrographs comparing the untreated and treated meshes (Figure 10). Low and high magnification images of pristine Optilene mesh LP (Figures 10a and 10d, respectively) show the smooth and fibrillary morphology of individual fibres, which present a diameter of $113.8 \pm 0.61 \mu \mathrm{m}$. When the PNIPAAm hydrogel is deposited with $\mathrm{GY}=0.50 \mathrm{mg} / \mathrm{cm}^{2}$, its presence above the monofilaments is evident (Figures 10b and 10e). The fibrillary morphology of the pristine polymer disappeared and a smooth material was deposited. For samples with the highest GY content $\left(3.89 \pm 0.12 \mathrm{mg} / \mathrm{cm}^{2}\right)$, the hydrogel is observed above the fibres and between them (Figures $10 \mathrm{c}$ and 10f). Moreover, despite the thickness of the hydrogel layer is high (Figure 10f), the adhesion to the fibres is excellent, as was proved by cryofracture SEM. The hydrogel does not detach from the fibre surface. Figure 11 displays the cross section of the iPP-g-PNIPAAm for fibres with the highest GY value. A network structure with a heterogeneous porosity is observed, the average diameter of pores being $66.2 \pm 5.8 \mu \mathrm{m}$
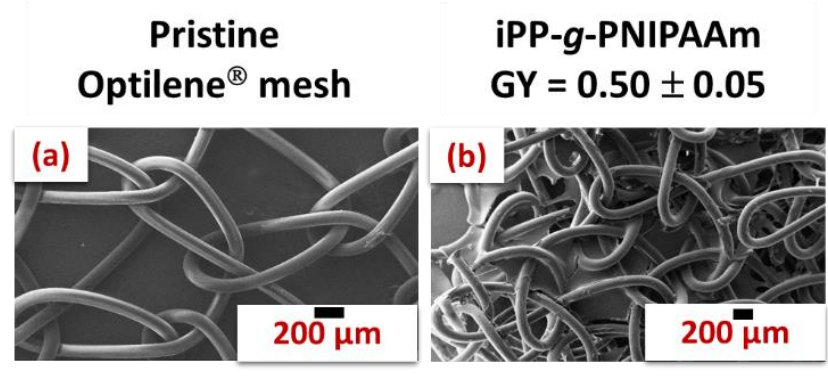

$$
\begin{aligned}
& \text { iPP-g-PNIPAAm } \\
& \text { GY = } 3.89 \pm 0.12
\end{aligned}
$$
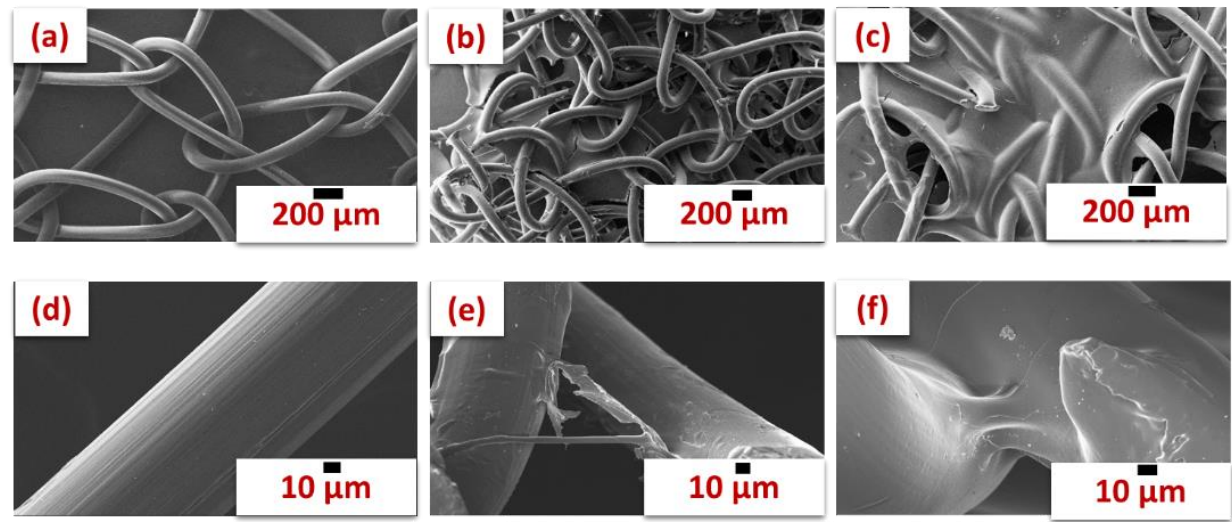

Increasing the GY

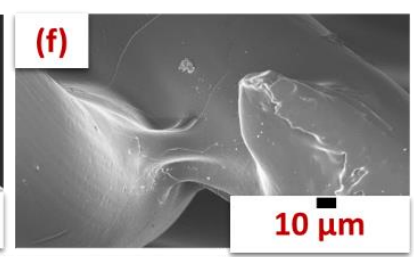

Figure 10. SEM micrographs of (a,d) pristine iPP mesh and (b,c,e,f) iPP mesh-g-PNIPAAm samples with different GY values: (b,e) $0.50 \pm 0.05$ mg/cm² and (c,f) $3.89 \pm 0.12$ $\mathrm{mg} / \mathrm{cm}^{2}$.

\section{Gel morphology}
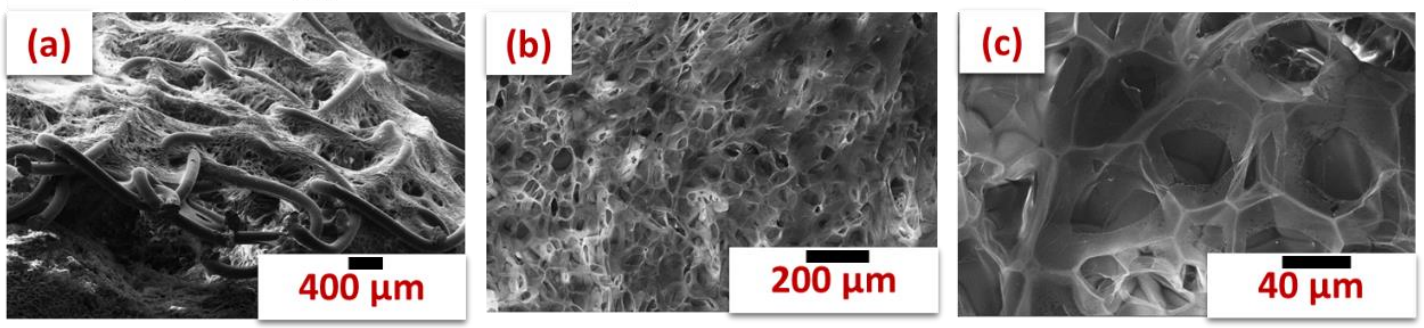

Figure 11. SEM micrographs of iPP- $g$-PNIPAAm sample with $\mathrm{GY}=3.89 \pm 0.12 \mathrm{mg} / \mathrm{cm}^{2}$ that certifies the porous inner structure of the PNIPAAm hydrogel. The crosssection images were taken after cryo-fracture of previously lyophilised Optilene ${ }^{\circledR}$ mesh fibres in liquid nitrogen.

Finally, once established the best plasma functionalization conditions and the best GY result, the volume phase transition temperature (VPTT) and the thermoresponsiveness of the grafted PNIPAAm hydrogels have been studied (Figure 12). The iPP-g-PNIPAAm bilayer system (Figure 12a) was soaked in distilled water and the variation of the chain length was evaluated between 24 and $40^{\circ} \mathrm{C}$. The VPTT was evaluated at the initial turning point in the graph reported in Figure $12 \mathrm{~b}$. A value very similar to the typical low critical solution temperature (LCST) of PNIPAAm of about $32^{\circ} \mathrm{C}$ was found, being in this case $33.2^{\circ} \mathrm{C}$. Below the VPTT, the hydrogel is hydrophilic and swollen in water; in contrast, above the VPTT, it becomes hydrophobic and decrease in volume ${ }^{33}$. The bilayer system was found to be completely flexible, despite the highest weight PNIPAAm hydrogel $\left(3.89 \pm 0.12 \mathrm{mg} / \mathrm{cm}^{2}\right)$ was employed for this step. 

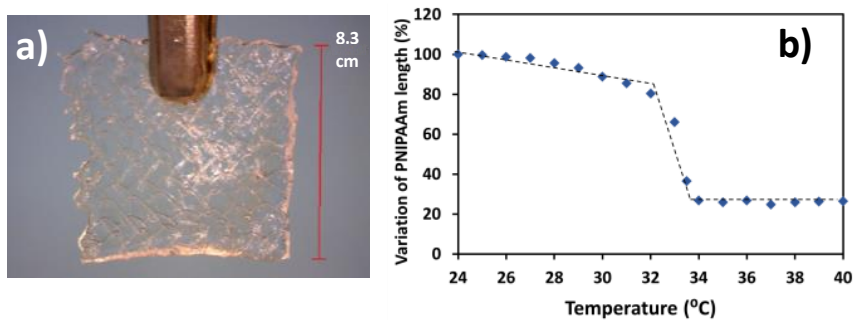

Figure 12. (a) Digital photograph of the new iPP-g-PNIPAAm thermoresponsive surgical mesh, and (b) variation of PNIPAAm length as a function of temperature, measured by optical microscopy (VTTP behaviour).

\section{Conclusions}

The architecture of commercial surgical meshes usually rises difficult issues for coating. In the present study, the covalent bonding of PNIPAAm thermosensitive hydrogel and non-absorbable polypropylene meshes has been successfully achieved. The process starts with the generation of $\mathrm{C}-\mathrm{O}, \mathrm{C}-\mathrm{OH}$ and $\mathrm{O}-\mathrm{C}=\mathrm{O}$ active groups onto iPP surface, after controlled plasma discharge. Unexpectedly, the amount of such active groups, increase considerably by doubling the power plasma discharge and maintaining the purging pressure low, i.e. regulating the ratio of $\mathrm{O}_{2}$ /air inside the reactor. In fact, the amount of PNIPAAm generated after plasma activation at $250 \mathrm{~W}$, which has been expressed in terms of graft yield, is four times the volume obtained after surface functionalization at 500W. The subtle augment of GY in the latter ones can be explained in basis of the relative concentration of $\mathrm{C}-\mathrm{OH}$ groups (very active for the grafting), which is twice higher for the sample treated at $250 \mathrm{~W}$ than for that treated at $500 \mathrm{~W}$. The mechanism for such covalent bonding between NIPAAm molecules and iPP functionalized surface was supported by XPS analysis, performed per triplicate, and evaluating each deconvoluted peak. Similar conclusions were reached by analysing the FTIR and micro-Raman absorption bands, with the advantage that such techniques are much less expensive and time-saving in comparison to XPS analysis.

Our results show that the PNIPAAm thermosensitive hydrogel is an excellent material to be covalently grafted to the iPP surface, after $\mathrm{O}_{2}$-plasma treatment, due to its good adherence and the thermosensitive response achieved after blending.

The main handicap to be overcome in future studies will be to control the PNIPAAm chains growth, which should occur onto the fibre surfaces only, avoiding plugging the mesh pores. For this purpose, surface functionalization and NIPAAm polymerization, both under atmospheric plasma conditions, would be an alternative to be envisaged.

\section{Conflicts of interest}

There are no conflicts to declare.

\section{Acknowledgements}

This work was supported by MINECO (MAT2015-69367-R) and the Agència de Gestió d'Ajuts Universitaris i de Recerca (2017SGR359). S. Lanzalaco acknowledges funding from the European Union's Horizon 2020 research and innovation programme under the Marie Sklodowska-Curie grant agreement № 796292 (Project 4D-POLYSENSE). Plasma equipment facilities were offered by B Braun Surgical S.A. (Spain). Support for the research by C. Alemán was received through the prize "ICREA Academia 2015" for excellence in research, funded by the Generalitat de Catalunya (Catalonia-Spain).

\section{References}

B. Bae, B. Chun and D. Kim, Polymer (Guildf)., 2001, 42, 7879-7885.

D. Garcia, O. Fenollar, R. Lopez, R. Sanchis and R. Balart, JournalofAppliedPolymerScience, 2008, 110, 1201-1207.

P. Slepička, A. Vasina, Z. Kolská, T. Luxbacher, P. Malinský, A. Macková and V. Švorčík, Nucl. Instruments Methods Phys. Res. Sect. B Beam Interact. with Mater. Atoms, 2010, 268, 2111-2114.

F. C. Usher, Am. J. Surg., 1959, 97, 629-633.

R. C. Read, Hernia, 1999, 3, 167-171.

S. Lanzalaco and E. Armelin, Gels, 2017, 3, 36.

D. Hetemi and J. Pinson, Chem. Soc. Rev., 2017, 46, 5701-5713. 
R. A. Wolf, Plast. Surf. Modif., 2010, 81-136.

C. Mühlhan, S. Weidner, J. Friedrich and H. Nowack, Surf. Coatings Technol., 1999, 116-119, 783-787.

S. Gorgieva, M. Modic, B. Dovgan, M. Kaisersberger-Vincek and V. Kokol, Plasma Process. Polym., 2015, 12, $237-251$.

N. Cui and N. Brown, Appl. Surf. Sci., 2002, 189, 1-8.

R. Molina, C. Ligero, P. Jovančić and E. Bertran, Plasma Process. Polym., 2013, 10, 506-516.

R. Nisticò, A. Rosellini, P. Rivolo, M. G. Faga, R. Lamberti, S. Martorana, M. Castellino, A. Virga, P. Mandracci, M. Malandrino and G. Magnacca, Appl. Surf. Sci., 2015, 328, 287-295.

C. Labay, J. M. Canal, M. Modic, U. Cvelbar, M. Quiles, M. Armengol, M. A. Arbos, F. J. Gil and C. Canal, Biomaterials, 2015, 71, 132144.

D. Hegemann and S. Gallen, Plasma Polymer Deposition and Coatings on Polymers, Elsevier, 2014, vol. 4.

M. J. Shenton and G. C. Stevens, J. Phys. D. Appl. Phys., 2001, 34, 2761-2768.

S. Roy and C. Y. Yue, Plasma Process. Polym., 2011, 8, 432-443.

K. S. Siow, S. Kumar and H. J. Griesser, Plasma Process. Polym., 2015, 12, 8-24.

F. Hilt, N. D. Boscher, D. Duday, N. Desbenoit, J. Levalois-Grützmacher and P. Choquet, ACS Appl. Mater. Interfaces, 2014, 6, 1841818422.

F. Loyer, G. Frache, P. Choquet and N. D. Boscher, Macromolecules, 2017, 50, 4351-4362.

J. Dai, X. Liu, Y. Xiao, J. Yang, P. Qi, J. Wang, Y. Wang and Z. Zhou, RSC Adv., 2015, 5, 71240-71252.

R. Molina, J. M. Teixidó, C. W. Kan and P. Jovančić, ACS Appl. Mater. Interfaces, 2017, 9, 5513-5521.

F. Loyer, A. Combrisson, K. Omer, M. Moreno-Couranjou, P. Choquet and N. D. Boscher, ACS Appl. Mater. Interfaces, 2019, 11, 13351343.

B. Sanz, C. von Bilderling, J. S. Tuninetti, L. Pietrasanta, C. Mijangos, G. S. Longo, O. Azzaroni and J. M. Giussi, Soft Matter, 2017, 13, 2453-2464.

Y. J. Kim and Y. T. Matsunaga, J. Mater. Chem. B, 2017, 5, 4307-4321.

P. Jovančić, A. Vílchez and R. Molina, Plasma Process. Polym., 2015, 13, 752-760.

K. Iwai, Y. Matsumura, S. Uchiyama and A. P. de Silva, J. Mater. Chem., 2005, 15, 2796.

C. Minogianni, K. G. Gatos and C. Galiotis, Appl. Spectrosc., 2005, 59, 1141-1147.

J. Martin, M. Ponçot, J. M. Hiver, P. Bourson and A. Dahoun, J. Raman Spectrosc., 2013, 44, 776-784.

T. Sundell, H. Fagerholm and H. Crozier, Polymer (Guildf)., 1996, 37, 3227-3231.

K. Zubik, P. Singhsa, Y. Wang, H. Manuspiya and R. Narain, Polymers (Basel)., 2017, 9, 1-17.

L. B. Peppas and R. S. Harland, Absorbent Polymer Technology; Elsevier: Amsterdam, The Netherlands, 1990.

A. Tiwari and B. Raj, Reactions and Mechanisms in Thermal Analysis of Advanced Materials., 2015.

G. S. Ghodake, N. G. Deshpande, Y. P. Lee and E. S. Jin, Colloids Surfaces B Biointerfaces, 2010, 75, 584-589.

G. Yang, J. Zhang, S. Zhang, L. Yu, P. Zhang and B. Zhu, Tribol. Int., 2013, 62, 163-170.

G. Yang, J. Zhao, L. Cui, S. Song, S. Zhang, L. Yu and P. Zhang, RSC Adv., 2017, 7, 7944-7953.

Y. C. Tyan, J. Der Liao, Y. Te Wu and R. Klauser, J. Biomater. Appl., 2002, 17, 153-178.

R. Morent, N. De Geyter, C. Leys, L. Gengembre and E. Payen, Surf. Interface Anal., 2008, 40, 597-600.

G. Kravets, L. I.;Elinson, V. M.; Ibragimov, R. G.; Mitu, B.; Dinescu, J. Phys. Conf. Ser., 2018, 982, 012011.

Y. C. Tyan, J. Der Liao, R. Klauser, I. Der Wu and C. C. Weng, Biomaterials, 2002, 23, 65-76.

R. M. Khafagy, J. Polym. Sci. Part B Polym. Phys., 2006, 44, 2173-2182.

D. E. Gen, K. A. Prokhorov, G. Y. Nikolaeva, E. A. Sagitova, P. P. Pashinin, B. F. Shklyaruk and E. M. Antipov, Laser Phys., 2011, 21, 125-129.

M. Arruebarrena de Baez, P. J. Hendra and M. Judkins, Spectrochim. Acta Part A, 1995, 51, 2117-2124. 